\title{
ANÁLISIS BIBLIOMETRICO DE LAS TESIS DOCTORALES ESPAÑOLAS EN ARTES MARCIALES Y PUBLICACIONES CIENTÍFICAS DE SUS AUTORES
}

\author{
ANÁLISE BIBLIOMÉTRICA DAS TESES DE DOUTORADO NA ESPANHA EM \\ ARTES MARCIAIS E PUBLICAÇÕES CIENTÍFICAS DE SEUS AUTORES
}

BIBLIOMETRIC ANALYSIS OF SPANISH DOCTORAL THESIS IN MARTIAL ARTS AND SCIENTIFIC PUBLICATIONS OF THEIR AUTHORS

Vicenç Hernández González*, Nuria Sans-Rosell *, Carme Jové-Deltell*, Joaquin Reverter-Masia *

\section{Palabras clave:} Tesis Académicas. Artes Marciales. Publicaciones periódicas como asunto. Bibliometría.
Palavras chave Dissertações Acadêmicas. Artes Marciais. Publicações Periódicas como Assunto. Bibliometria.
Resumo: el objetivo es verificar si la obtención del título de Doctor es un factor que promueve la formación de nuevos investigadores. Presentar el índice $\mathrm{h}$ de los doctoresautores así como la relación que hay con medidas utilizadas para evaluar la productividad investigadora, como número de artículos e índice de citas. Mediante la base de datos TESEO y Web of Science se obtuvieron los registros de trabajos. Los resultados mostraron un total de 86 tesis (2,2 tesis/año), más de 285 trabajos en Web of Science, con media de 5,81 trabajos/autor. La media de citas por trabajo es de 4,25, mientras que la de citas por autor se sitúa en 24,73. La media general de índice h es de 2,02 por autor. Se concluyen baja producción de tesis doctorales en España en Artes Marciales. Más de la mitad de los doctores-autores publican trabajos en Web of Science con notable difusión científica de sus tesis.
Resumo: o objetivo é verificar se a obtenção do título de Doutor é um fator que promove a formação de novos investigadores. Apresentar o índice $\mathrm{h}$ dos doutores-autores, bem como a relação que existe com as medidas utilizadas para avaliar a produtividade da pesquisa, como número de artigos e índice de citações. Mediante o banco de dados TESEO e a Web of Science obteve-se os registros do trabalho. Os resultados mostraram um total de 86 teses (2,2 teses/ano), mais de 285 trabalhos na Web of Science, com uma média de 5,81 trabalhos/autor. O número médio de consultas por trabalho é de 4,25, enquanto o número de consultas por autor é de 24,73. 0 índice geral h-index é 2.02 por autor. Concluímos baixa produção de teses de doutorado em Espanha em artes marciais. Mais de metade dos autores doutores-autores publicam trabalhos na Web of Science com notável difusão científica de suas teses.
Keywords: Academic dissertations. Martial Arts. periodicals as topic. Bibliometrics.
Abstract: the objective is to verify if obtaining the title of Doctor is a factor that promotes the formation of new investigators. Present of the $h$-index of the authors-authors as well as the relationship that exists with measures used to evaluate research productivity, such as number of articles and index of citations. The TESEO and Web of Science database obtained the work records. The results showed a total of 86 theses (2.2 theses / year) more than 285 works in Web of Science, with an average of 5.81 works / author. The average number of appointments per work is 4.25 , while the number of appointments per author stands at 24.73 . The overall mean $\mathrm{h}$-index is 2.02 per author. We conclude a low production of doctoral theses in Spain in Martial Arts. More than half of the doctor-authors publish works in Web of Science with a remarkable scientific diffusion of their theses.
*Universidad de Lleida. Lleida, España. E-mail: vicens_h_g@didesp.udl.cat n.sans@ didesp.udl.cat; carme.jove@ didesp.udl.cat: reverter@didesp.udl.cat

Recebido em: 26-10-2017 Aprovado em: 02-03-2018

DOI: http://dx.doi.org/10.22456/1982-8918.77660 (c) (1) (3) Licence 


\section{INTRODUCCIÓN}

Una tesis doctoral es la culminación del ciclo de aprendizaje de un científico en proceso de formación bajo la dirección de uno o varios doctores que hacen las funciones de directores de tesis (Fuentes; Arguimbau, 2010). Una vez superada la defensa pública, el doctorando obtiene el título de doctor y, a partir de ese momento, tiene la posibilidad de difundir el conocimiento (artículos, comunicaciones, etc.).

El estudio de las tesis doctorales como objeto de análisis es enormemente provechoso ya que éstas constituyen una de los mejores espejos donde se reflejan las tendencias de la investigación de las universidades. Además, nos permite analizar no solo la evolución científica de un área concreta de estudio sino también identificar cuáles son sus principales protagonistas (Repiso; Torres; Delgado, 2011).

Numerosos trabajos en España, han analizado la producción y difusión científica a través de las tesis doctorales, Fuentes y Arguimbau (2010) analizan las tesis doctorales aprobadas en España durante el período 1997-2008 de dieciocho campos científicos distintos, concluyendo la buena capacidad del sistema académico español para formar nuevos investigadores. Además, existen estudios sobre las siguientes área temáticas: Educación física (Ponce de León Elizondo; Gargallo; Loza, 1998); Medicina(Centeno et al., 2001; Figueredo, et al.,2002; Diego; Prim, 2008); Educación Matemática (CURIEL-MARÍN; Fernández-CANO, 2015); Psicología (Agudelo et al., 2003; Olivas-Ávila; Musi-Lechuga, 2010); Feminismo (Torres; Torres, 2005); Relaciones Públicas (Castillo; Xifra, 2006); Televisión (Repiso; Torres; Delgado, 2011) entre otros.

Paralelamente al estudio de las tesis doctorales, también se han hecho estudios bibliométricos sobre revistas científicas (Alcaín; Carpintero, 2001; Albacete-Sáez; FuentesFuentes, 2010; Reverter-Masia et al., 2015), o mediante el análisis de la producción científica a partir de trabajos publicados en Web of Science (Sierra; Zubeidat, 2002; Carretero-Dios; De los Santos-Roig; Buela-Casal, 2005; Reverter-Masia et al., 2013; Devís-Devís; Villamón; Valenciano-Valcárcel, 2014; Hernández-González et al., 2016).

Hoy por hoy, las publicaciones se emplean como medida de la competencia y el éxito profesional de éstos autores y de los organismos que representan (Claxton, 2005). Tanto las instituciones como los investigadores que en ellas trabajan necesitan obtener recursos económicos, promocionarse y un reconocimiento público y ello dependerá de la cantidad y la calidad de sus publicaciones (SALGADO; PAÉZ, 2007).

En la actualidad, los indicadores bibliométricos más utilizados se basan en el recuento de publicaciones, especialmente el de artículos científicos, indicador bibliométrico crucial para determinar la productividad, visibilidad, impacto de la actividad científica (DELGADO LÓPEZCÓZAR; JIMÉNEZ-CONTRERAS; RUIZ-PÉREZ, 2009; BOLAÑOS, 2012).

Tomando como premisa el concepto de que el producto final de una investigación científica es la publicación de un artículo en revistas de relevancia, hemos analizado la capacidad investigadora de los autores de las tesis, en función del recuento de sus publicaciones en revistas incluidas en la base de datos Web of Science.

El objetivo del presente estudio es verificar si la obtención del título de Doctor, es un factor que promueve la formación de nuevos investigadores a través de la publicación de trabajos en Web of Science. En segundo lugar, presentar el índice h de los doctores para examinar la 
relación que hay entre el índice h y otras conocidas medidas utilizadas en España para evaluar la productividad investigadora, como el número de artículos e índice de citas.

\section{MATERIAL Y MÉTODO}

\subsection{Diseño}

Se trata de un estudio descriptivo, comparativo y correlacional de análisis de documentos. Se tomó en cuenta las consideraciones propuestas por Hirsch (2005), así como las propuestas por Ramos-Álvarez et al., (2008) y las recogidas en los Principios de Berlín para Rankings de Instituciones de Educación Superior (INTERNATIONAL RANKING EXPERT GROUP, 2006) y el estudio de Olivas-Ávila y Musi-Lechuga (2010).

\subsection{Unidad de análisis}

- Fichas de Tesis Doctorales: se emplearon los datos registrados en la base de datos Tesis Españolas Ordenadas (TESEO).

- Base de datos (ISI) Web of Knowledge. Ésta se encuentra disponible a través de la plataforma (ISI) Web of Knowledge.

\subsection{Procedimiento}

\subsubsection{Tesis doctorales sobre artes marciales}

La base de datos TESEO del Consejo de Universidades, recoge y permite recuperar información acerca de las tesis doctorales leídas y consideradas aptas en todas las Universidades españolas desde 1976. Dicho fichero seencuentra disponibleen Internet $<$ https://www.educacion. gob.es/teseo/irGestionarConsulta.do;jsessionid=169BFE558405DAEB751ACE2600B92F09>.

Se accedió a él utilizando la estrategia de búsqueda: 1) palabras claves: "artes marciales"; "Lucha”; “Judo"; "Karate”, "taekwondo"; "aikido", "ju-jutsu”, "combate”, "kickboxing"; "boxeo"; "muay-thai"; "esgrima", "kendo"; "wushu"; "full-contact"; "kung-fu”, en el título o en el resumen de la tesis.

A partir de la búsqueda se obtuvieron los registros con los títulos de las tesis realizadas hasta febrero de 2017. Cada uno de ellos permitía el acceso a un archivo de texto con información ampliada (autor-doctor, director, título, resumen, Universidad, Facultad, centro de realización, curso lectivo y descriptores), se recopiló y trasladó a una base de datos que denominamos "Tesis sobre artes marciales" (Microsoft Excel 2010).

\subsubsection{Descriptores}

Al estudiar los descriptores que obtuvimos de las citadas bases de datos, apreciamos que cada tesis se encuadraba en diferentes descriptores y que éstos no eran excluyentes entre sí. Por tanto, se procedió a determinar un solo descriptor para cada una de las tesis. Para una revisión más detallada del proceso es importante referirse al estudio de Ponce de León, Gargallo y Loza (1998). 


\subsubsection{Artículos y revistas publicados por los autores de tesis doctorales}

En primer lugar, se seleccionaron los participantes a partir de la información obtenida de la base de datos TESEO. En segundo lugar, los datos obtenidos se realizaron en función de los registros recabados en la (ISI) Web of Knowledge, teniendo en cuenta que en muchas ocasiones hay errores en los nombres de los autores. Se utilizó la siguiente estrategia de búsqueda: 1) primer apellido + primera inicial de sus nombres; 2) primer apellido + segundo apellido + primera inicial de sus nombres. Debido a que en algunos casos de apellidos de uso muy extendido en España existía coincidencia de apellidos e iniciales en autores que podían ser distintas personas, en estos casos empleamos adicionalmente otras estrategias para circunscribir la búsqueda: a) empleo de la segunda inicial; b) coincidencia de los títulos de los artículos con la especialidad o con el tema de la tesis; c) coincidencia de la filiación del autor con la localización de la universidad donde se realizó la tesis; d) coincidencia entre los coautores y otros miembros del mismo departamento. También se emplearon truncadores y operadores booleanos para garantizar la obtención del total de artículos por autor.

El proceso de recolección de la información se realizó en la base de datos mencionada, ingresando en el apartado de "General Search», en donde se utilizó el campo de "Author» y «Address», incluso en algunos casos se utilizó el campo «Topic».

\subsubsection{Método de cálculo Índice h}

El método de cálculo del Índice $h(\mathrm{HIRSCH}, 2005)$ y descrito con más detalle por Imperial y Rodríguez-Navarro(2005) consiste en los siguientes pasos: (1) Entrar en Web of Knowledge y elegir la opción "General Search»; (2) escribir el nombre del autor en la ventana correspondiente; (3) cuando el sistema responda la lista de publicaciones, utilizar la opción de clasificación por orden de citas ( Sortby times cited ); y (4) a continuación identificar el último trabajo cuyo número de orden sea superior al del número de citas que tiene asociado. Ese número de orden corresponde al Índice $h$ del autor.

\subsubsection{Número total de citas y cita máxima}

Las búsquedas se llevaron a cabo durante los meses de enero y febrero de 2017 por dos investigadores de manera independiente con la finalidad de obtener mayor fiabilidad de los resultados.

Los datos obtenidos, fueron trasladados a una base de datos que denominamos "autores-doctores" y que subdividimos en tantos subarchivos como autores-doctores teníamos. En cada archivo compilamos los datos extraídos de (ISI) Web of Knowledge, autores y título del artículo, revista donde fue publicado, año de publicación, número de citas del trabajo, número de coautores, índice $h$ del autor-doctor.

\subsubsection{Análisis de los datos}

Se reordenó la base de datos "Tesis sobre Artes Marciales" según distintos criterios, que nos permitió obtener datos generales referentes a: directores, universidades, facultades y año en el que el doctorando presentó su tesis. 
Con los datos numéricos de las publicaciones individuales de cada uno de los autores se confeccionó una tabla global: 1) la cantidad total de publicaciones de cada autor con sus correspondientes títulos; 2) las revistas donde se publicaron; 3) número de citas de cada trabajo; 4) año de publicación; 5) número de coautores; 6) índice h Hirsch del autor.

Los datos de las distintas variables se cruzaron entre sí, y en los casos en los que se consideró apropiado, se realizó un análisis estadístico mediante correlaciones, estableciéndose como significativa una $p<0,0001$.

\section{RESULTADOS}

\subsection{Productividad de las tesis}

desde el año 1976 hasta febrero de 2017 se habían incorporado a la base de datos TESEO 86 tesis doctorales que cumplían nuestro criterios de búsqueda; esta cifra representa un promedio de 2,2 tesis por año (figura 1).

Figura 1 - Evolución de la productividad en número de Tesis doctorales

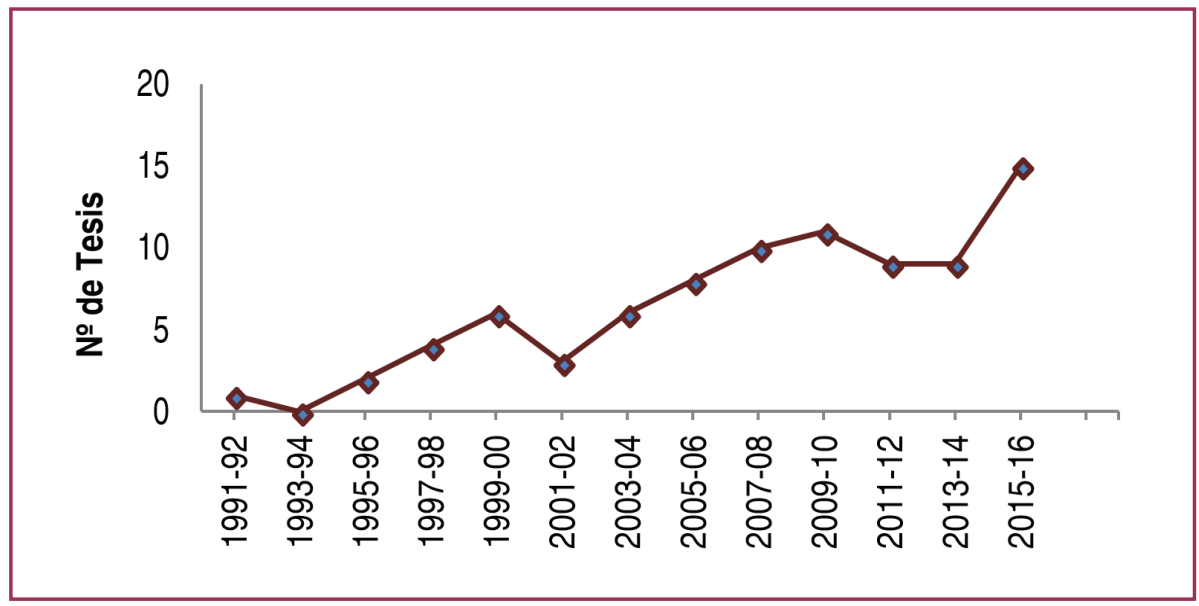

Fuente: elaboración propia

Se denota el crecimiento progresivo característico de una disciplina que se encuentra en fase de desarrollo. Se puede observar un crecimiento exponencial a partir del año 200102.

En la tabla 1, se muestra a los 10 directores más productivos en número de tesis doctorales dirigidas.

Los directores con 1 tesis dirigida dirigen el $68 \%$ de las tesis, mientras que el $5,12 \%$ de los directores más productivos dirigen el $15,17 \%$ de las tesis.

La tabla 2, muestra las 10 universidades donde se leyeron más tesis. Las 86 tesis doctorales fueron defendidas en 32 universidades distintas.

La universidad más productiva en número de tesis es la Univ. Palmas de Gran Canaria $(10,4 \%)$, seguida de la Univ. de León $(9,3 \%)$ y la Univ. de Castilla la Mancha $(8,1 \%)$. 
Tabla 1 - Relación de los 10 directores más productivos.

\begin{tabular}{lcc}
\hline Dirección & $\mathrm{f}$ & $\%$ \\
\hline Amador Ramírez, Fernando & 5 & 10,87 \\
García García, José Manuel & 4 & 8,70 \\
Padial Puche, Paulino & 4 & 8,70 \\
CarratallaDeval, Vicent & 3 & 6,52 \\
Sánchez Bañuelos, Fernando & 3 & 6,52 \\
Saucedo Morales, Francisco & 3 & 6,52 \\
Álvarez del Palacio, Eduardo & 2 & 4,35 \\
FericheFernández-Castanys, M. Belén & 2 & 4,35 \\
Garces Martín, Gerard & 2 & 4,35 \\
García Pallares, Jesús & 2 & 4,35 \\
\hline
\end{tabular}

$f=$ frecuencia; $\%$ = porcentaje sobre los directores más productivos

Fuente: elaboración propia

Tabla 2 - Productividad institucional.

\begin{tabular}{llc}
\hline Universidad & f & $\%$ \\
\hline Univ. de Las Palmas de Gran Cana. & 9 & 10,47 \\
Univ. de León & 8 & 9,30 \\
Univ. de Castilla-La Mancha & 7 & 8,14 \\
Univ. de Barcelona & 6 & 6,98 \\
Univ. de Granada & 6 & 6,98 \\
Univ. Politécnica de Madrid & 6 & 6,98 \\
Univ. de València (Estudi Gene.) & 5 & 5,81 \\
Univ. de A Coruña & 4 & 4,65 \\
Univ. Autónoma de Madrid & 3 & 3,49 \\
Univ. de Murcia & 3 & 3,49 \\
\hline
\end{tabular}

Fuente: elaboración propia

Figura 2 - Descriptores más utilizados en las tesis doctorales de artes marciales.

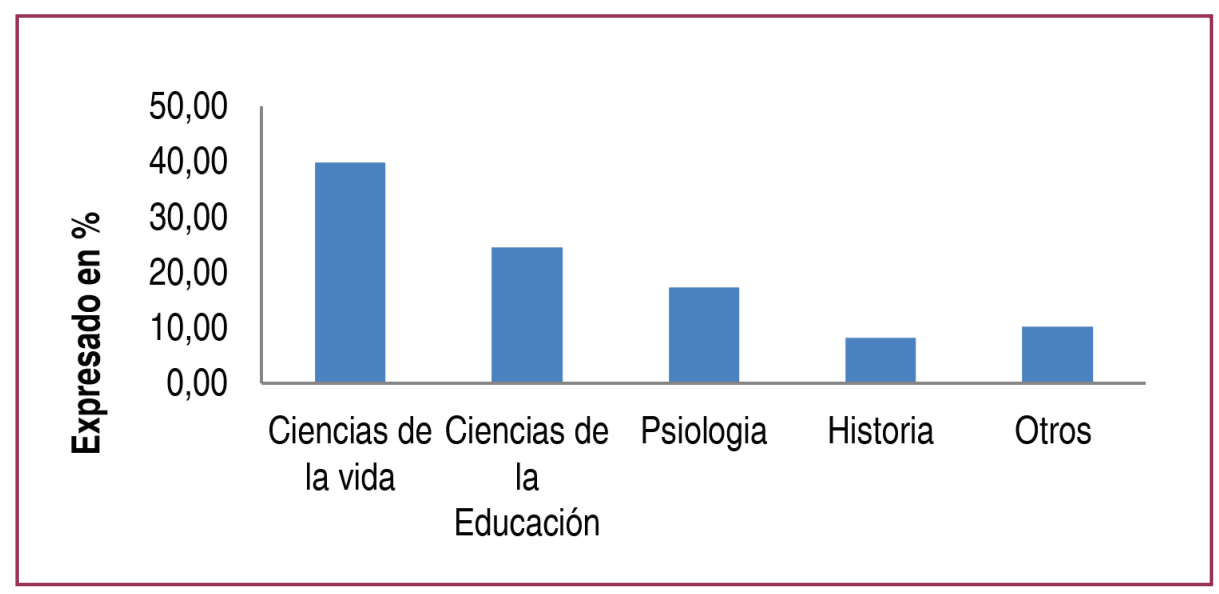

Fuente: elaboración propia 
Atendiendo al campo de estudio, podemos hacer referencia principalmente a 3 campos de estudios, el ámbito de las Ciencias de la Vida con el $40 \%$ del total, seguida de las Ciencias de la Educación con más del 20\% y la psicología que se sitúa alrededor del 18\%.

\subsection{Productividad de los distintos indicadores de calidad}

La producción científica de los autores de las tesis medidas en número de artículos, citas recibidas por sus trabajos publicados e índice $\mathrm{h}$ de los autores-doctores se muestran en la tabla 3.

Tabla 3 - Datos de productividad mediante número de artículo, número de citas e índice h.

\begin{tabular}{ccc}
\hline $\begin{array}{c}\text { № total de tesis } \\
\text { defendidas }\end{array}$ & $\begin{array}{c}\text { Producción } \\
\text { total }\end{array}$ \\
\hline & № artículos en WoS & 285 \\
& № autores que publican en WoS & 49 \\
Media artículos por autor & 5,81 \\
86 & № de citas totales & 1212 \\
& Media de citas por artículo & 4,25 \\
& Media de citas por autor & 24,73 \\
& № profesores con índice h & 48 \\
& Índice $\mathrm{h}$ total & 97 \\
& Media de índice h & 2,02 \\
& Media de autores por artículo & 5,18 \\
\hline
\end{tabular}

Fuente: elaboración propia

Se puede observar cómo de los 86 autores que defendieron su tesis doctoral, 49 de ellos, es decir el $56,9 \%$ publican trabajos en WoS, en total 285 trabajos. La media de trabajos por autor se sitúa en 5,81. Observando el índice de citación, recibieron un total de 1212, la media de citas por trabajo es de 4,25 y la media de citas por autor se sitúa en 24,73 citas.

Si atendemos al índice $h$, podemos observar como casi la totalidad de los autores que publican trabajos en WoS tienen índice h (48/49). Analizando la media de autores firmantes por trabajo, ésta se sitúa en 5,18 autores/trabajo.

Los trabajos son publicados en una gran variedad de revistas, en total 108 revistas. Las revistas donde más frecuentemente publicaban sus trabajos eran en primer lugar la Journal of Strength and Conditioning Research con 24 trabajos (representa el 14,81\%), seguida de la revista Archives of Budo con 16 trabajos, (representa el 9,88\%).

Las veinte revistas de la tabla 4 publican el $56,84 \%$ de los trabajos, mientras que el restante $43,16 \%$ de los trabajos están publicados en las 88 revistas que tienen publicado 102 trabajos como máximo.

Si analizamos los cuartiles de las revistas publican sus trabajos en mayor medida, observamos cómo el $21 \%$ de los trabajos están publicados en revistas del primer cuartil (4 revistas), $35,5 \%$ en revistas del segundo cuartil (5 revistas), 17,1\% en el tercer cuartil (3 revistas) y el $26,3 \%$ en revistas de cuarto cuartil ( 6 revistas). 
Tabla 4 - Ranking de las 20 revistas más con mayor número de trabajos publicados.

\begin{tabular}{lccc}
\hline \multicolumn{1}{c}{ Revistas } & f & $\%$ & Q \\
\hline Journal of Strength and Conditioning Research & 24 & 14,81 & Q2 \\
Archives of Budo & 16 & 9,88 & Q3 \\
Journal of Sports Sciences & 13 & 8,02 & Q2 \\
Plos One & 13 & 8,02 & Q1 \\
Revista de Psicología del Deporte & 13 & 8,02 & Q4 \\
International Journal of Sports Physiology and Performance & 9 & 5,56 & Q1 \\
European Journal of Sport Science & 8 & 4,94 & Q2 \\
Rev. Int. de Medicina y Ciencias de la Act. Física y del Deporte & 7 & 4,32 & Q4 \\
International Journal of Sports Science \& Coaching & 6 & 3,70 & Q4 \\
Journal of Human Kinetics & 6 & 3,70 & Q4 \\
Medicine and Science in Sports and Exercise & 6 & 3,70 & Q1 \\
Motricidad. European Journal of Human Movement & 5 & 3,09 & - \\
International Journal of Performance Analysis in Sport & 5 & 3,09 & Q3 \\
International Journal of the History of Sport & 5 & 3,09 & Q2 \\
Journal of Sports Science and Medicine & 5 & 3,09 & Q3 \\
Sport TK- Revista Euroamericana de Ciencias del Deporte & 5 & 3,09 & - \\
International Journal of Sports Medicine & 4 & 2,47 & Q1 \\
Journal of the International Society of Sports Nutrition & 4 & 2,47 & Q2 \\
Movimento & 4 & 2,47 & Q4 \\
Perceptual and Motor Skills & 4 & 2,47 & Q4 \\
\hline
\end{tabular}

$f=$ frecuencia; $\%=$ porcentaje sobre las veinte revistas; $Q=$ cuartil Fuente: elaboración propia

Tabla 5 - Ranking de las 20 revistas más con mayor número de citas en WoS.

\begin{tabular}{lccc}
\hline \multicolumn{1}{c}{ Revistas } & $\mathbf{f}$ & $\%$ & $\mathbf{Q}$ \\
\hline Journal of Strength and Conditioning Research & 166 & 13,70 & Q2 \\
Journal of Sports Sciences & 115 & 9,49 & Q2 \\
Journal of Biomechanics & 84 & 6,93 & Q2 \\
Plos One & 70 & 5,78 & Q1 \\
International Journal of Sports Physiology and Performance & 60 & 4,95 & Q1 \\
European Journal of Applied Physiology & 59 & 4,87 & Q1 \\
Medicine and Science in Sports and Exercise & 59 & 4,87 & Q1 \\
Revista de Psicología del Deporte & 52 & 4,29 & Q4 \\
Archives of Budo & 52 & 4,29 & Q3 \\
Journal of the International Society of Sports Nutrition & 37 & 3,05 & Q2 \\
Amino Acids & 33 & 2,72 & Q2 \\
British Journal of Sports Medicine & 28 & 2,31 & Q1 \\
Cultura y Educación & 28 & 2,31 & Q4 \\
Journal of Human Kinetics & 27 & 2,23 & Q4 \\
International Journal of Sports Medicine & 25 & 2,06 & Q1 \\
Journal of Sports Science and Medicine & 21 & 1,73 & Q3 \\
European Journal of Sport Science & 20 & 1,65 & Q2 \\
International Review for the Sociology of Sport & 19 & 1,57 & Q2 \\
International Journal of Obesity & 17 & 1,40 & Q1 \\
Clinical Physiology and Functional Imaging & 15 & 1,24 & Q3 \\
\hline
\end{tabular}


Podemos comprobar como la Journal of Strength and Conditioning Research es la revista que más citas recibe (tabla 5). En total, las veinte revistas que aparecen en el ranking, reciben 987 citas del total de 1212 citas, lo que supone el 81,44\% del total de citas. Matizar que, de las veinte revistas con mayor número de trabajos publicados, 12 de ellas son también las que reciben más citas. Correlacionando el número de trabajos publicados por el número de citas recibida, podemos observar una correlación positiva (.81).

Las revistas con más citas, son del primer y segundo cuartil $(67,2 \%)$. Únicamente 3 revistas son del tercer cuartil (15\%), con el 8,9\% de las citas, seguidas de las tres revistas restantes que son del cuarto cuartil (15\%) y que reciben el $10,8 \%$ de las citas.

\subsection{Productividad de los distintos indicadores de calidad derivados de las tesis doctorales}

La figura 3, presenta una distribución bianual de los trabajos publicados en WoS por los doctores-autores. La publicación de estos trabajos empezó en el año 1991, pero es a partir del año 2009-2010 cuando se consolida con una producción significativa de 37 trabajos.

Figura 3 - Evolución de la productividad en número trabajos publicados en Web of Science.

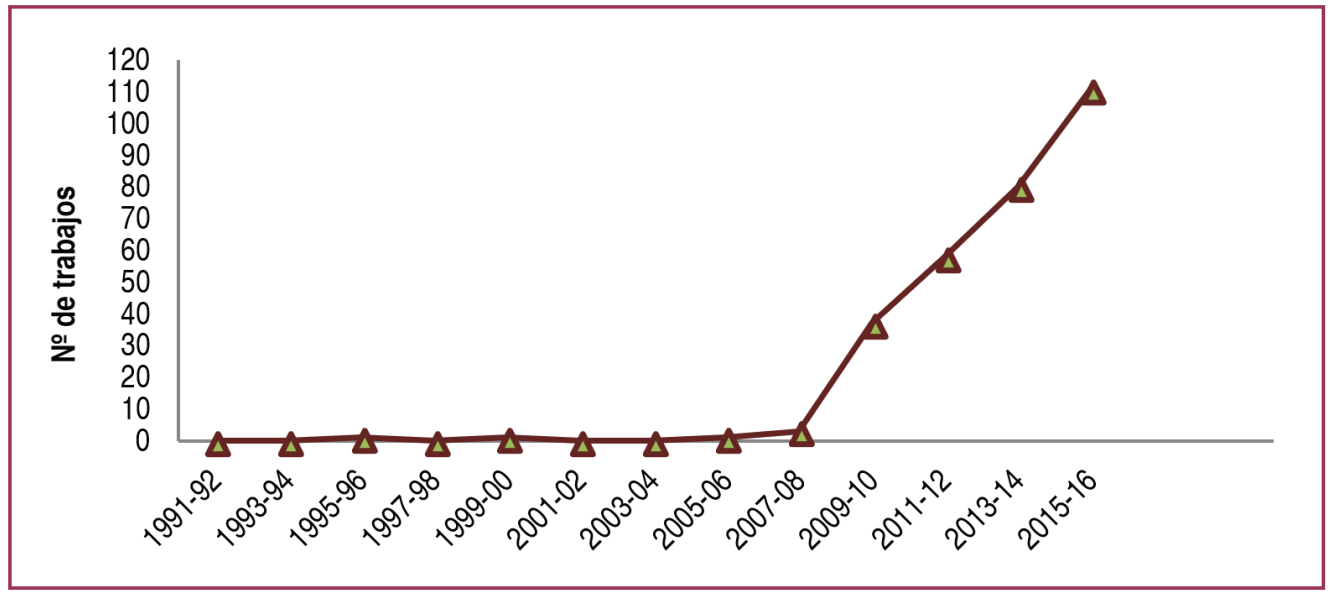

Fuente: elaboración propia

\subsection{Convergencia entre los indicadores de la productividad y calidad científica}

De acuerdo con los objetivos, si atendemos a la relación del Índice $h$ con el total de citas de cada autor, hemos podido observar que la correlación es positiva 0.923 , y lo mismo ocurre con la correlación del Índice $h$ con el número de artículos por investigador 0.862 y el número de artículos y número de citas 0.93 . En todos los casos la magnitud de la correlación es muy elevada (tabla 6).

Tabla 6 - Correlaciones entre Índice $h$, № de citas (NC) y № de artículos (NA) en WoS.

\begin{tabular}{|c|c|c|}
\hline \multicolumn{3}{|c|}{ CORRELACIONES } \\
\hline Índice $\mathrm{h}$ x NA & Índice $\mathrm{h} \times \mathrm{NC}$ & $N A \times N R$ \\
\hline $0,8626^{*}$ & $0,923^{*}$ & $0,9302^{*}$ \\
\hline
\end{tabular}




\section{DISCUSIÓN}

Este es el primer estudio que se tenga conocimiento, que analiza la producción científica a partir de las tesis doctorales en las ciencias del deporte en España y más concretamente en el ámbito de las artes marciales. Con anterioridad, autores como Ponce de León, Gargallo y Loza (1998) ya presentaron un trabajo donde analizaban las tesis doctorales en Educación Física, centrado en los descriptores. Por su parte, Ortega et al., (2015) presentaron un estudio dónde analizaban el papel de la mujer en las tesis doctorales de ciencias del deporte.

Paralelamente a estos trabajos, otros autores han analizado en el ámbito de las ciencias del deporte, la producción científica a partir de la publicación de artículos (DEVÍSDEVÍS; VILLAMÓN; VALENCIANO-VALCÁRCEL, 2014; PALAZÓN; ORTEGA; GARCÍAANGULO, 2015; HERNÁNDEZ-GONZÁLEZ; REVERTER-MASIA; JOVÉ-DELTELL, 2017).Tal y como señalan Figueredo et al., (2002) este tipo de trabajos resultan útiles para conocer la realidad investigadora de una especialidad en un territorio determinado.

Nuestro estudio identifica 86 tesis doctorales sobre artes marciales. Entre los años 2007/2016 fueron defendidas la mayor parte de las tesis, auspiciadas sin duda, por el enorme crecimiento de la capacidad de la universidad española para generar conocimiento científico (REPISO; TORRES; DELGADO, 2011). El promedio de tesis anuales sobre Artes Marciales realizadas en nuestro país en los últimos 38 años, puede considerarse como muy bajo, aunque bien es cierto que las Artes Marciales representan a una parte muy pequeña de una disciplina más importante en nuestro país como es el de las Ciencias de la Actividad Física y del Deporte. Un dato positivo es que se observa una tendencia creciente en la publicación de tesis a partir del año 2006.

Los estudios de Moreno-Fernández y Moreno-Crespo (2016) concluyen que aquellos directores con al menos 4 trabajos de investigación dirigidos poseen una trayectoria relevante como director o directora de trabajos de investigación. En nuestro estudio, destacan Fernando Amador profesor de la Universidad de las Palmas de Gran Canaria, José Manuel García profesor de la Universidad de Castilla la Mancha y Paulino Padial Puche profesor de la Universidad de Granada como directores relevantes. En cambio, Fernández Cano et al., (2003) hablan de grandes directores cuando han dirigido más de 10 tesis. Por tanto, destacamos la ausencia de grandes directores (más de diez tesis dirigidas) dentro del ámbito de las artes marciales.

Las universidades con mayor producción de tesis son la Universidad de las Palmas de Gran Canaria, seguida de la Universidad de León. Según señala Price (1986), las instituciones pueden clasificarse en tres grupos: grandes productoras (más de 10 tesis), productoras medias (2-9 tesis) y productoras ocasionales (1 tesis). En nuestro estudio, no hay ninguna universidad con más de 10 trabajos defendidos. Una posible explicación puede ser la corta trayectoria científica que tiene esta disciplina en comparación por ejemplo con Anestesiología (FIGUEREDO et al., 2002); la educación matemática (FERNÁNDEZ CANO et al., 2003); los estudios de la mujer (TORRES; TORRES, 2005); la psicología (OLIVAS-ÁVILA; MUSI-LECHUGA, 2010); o la televisión (REPISO; TORRES; DELGADO, 2011).

Analizando los descriptores, pudimos observar como aquellas tesis doctorales que ubican sus descriptores en la categoría de Ciencias de la Vida, principalmente centran sus temas de estudio sobre aspectos relacionados con la adaptación del organismo al esfuerzo, la biomecánica, la antropometría, etc. Cabe reseñar que el entrenamiento deportivo sigue siendo 
la principal temática de las tesis defendidas, relegando en un segundo lugar las centradas en el aprendizaje de los deportes de combate.

Tal y como señalan Devís-Devís et al., (2010) las Ciencias de la Actividad Física, y por tanto las Artes Marciales, se ha valido de diferentes ramas del saber para la construcción de su propio conocimiento científico, no es de extrañar por tanto, que descriptores que son más propios de otras ramas científicas (como la medicina) sean utilizados en tesis doctorales del ámbito de las Artes Marciales

Aproximadamente la mitad de los doctores han publicado algún trabajo en revistas de reconocido prestigio, algo que sin duda es muy relevante para la construcción de un campo de investigación minoritario. Estos resultados son muy similares a los encontrados por Figueredo et al., (2002); o Reverter-Masia et al., (2013).

La media de trabajos publicados por autor se sitúa en 5,81. Estos resultados son algo inferiores a los encontrados por Olivas-Ávila y Musi-Lechuga (2010) en otras áreas de conocimiento, como en Psicología Social $(8,11)$ y Psicología Evolutiva y de la Educación $(6,64)$. Esta producción algo inferior a otras disciplinas podría deberse a que actualmente únicamente existe una revista especifica de impacto la Archives of Budoque publica sobre las Artes Marciales.

Mediante dicho trabajo, se ha pretendido sondear cual es el potencial del Índice $h$ como un indicador de la calidad de la producción científica de los doctores-autores en Artes Marciales. Si hacemos una comparación del Índice h con áreas afines en España (SALGADO; PÁEZ, 2007; REVERTER-MASIA et al., 2013) podemos constatar que el Índice $h$ de los autoresdoctores en nuestro estudio es aun relativamente bajo, una posible razón sea el corto período de tiempo que estos investigadores llevan publicando trabajos, prácticamente se inician a partir del año 2008. Tal y como señalan Gil Soares y Sardinha (2011), actualmente es necesario un trayecto de largo recorrido para semejarse al tamaño del índice en otros países y otras áreas de conocimiento.

La evolución temporal de la producción científica de los doctores-autores tiene un crecimiento exponencial, posiblemente este incremento sea debido a las políticas de las distintas agencias evaluadoras, tanto de profesorado como de instituciones.

Existen grandes diferencias en el rango de citas entre las disciplinas científicas. Las citas tienden a crecer hasta los 3-5 años en ciencias naturales y hasta los 5-7 años en ciencias sociales. Por ejemplo, el total de citas necesarias para estar en el 1\% de los artículos más citados oscilaba entre 285 citas para Biología Molecular y 32 en Educación según el ISI (1999). En nuestro estudio la media de citas es de 24,73 , por lo tanto los datos estarían por debajo de los señalados por ISI (1999) en ciencias de la educación.

La media de autores por artículo se sitúa en $5 \pm$, resultados similares a los encontrados por Reverter-Masia et al., (2015). El número de autores junto con la accesibilidad, se han señalado como factores predictores del grado de citación (CALVER; BRADLEY, 2010).

Si analizamos las revistas, se observa una gran concentración de trabajos en un grupo muy reducido de revistas. Además, no se observan diferencias significativas entre el cuartil de las revistas. Resultados muy diferentes a los encontrados por Reverter-Masia et al., (2015), donde observaron una tendencia de los profesores de Educación Física a publicar en revistas 
del tercer y cuarto cuartil. Por su parte, Jiménez-Contreras, Robinson-García y Cabezas-Clavijo (2011) en su estudio sobre producción e impacto científico de las diversas áreas científicas, concluyeron, por ejemplo, que en las ciencias sociales, únicamente el $9 \%$ publicaba en revistas del primer cuartil, en ciencias de la educación se situaba en el $10 \%$.

Prácticamente la totalidad de las citas son para un número muy reducido de revistas que son a la vez las que se sitúan en el primer y segundo cuartil, razón por lo que se podría afirmar que los autores usan esas revistas como fuente de consulta, que son las que después citan en sus artículos. Parece ser que el impacto es un indicador clave en la estrategia de los investigadores a la hora de seleccionar las revistas para citar (GONZÁLEZ DE DIOS et al., 2011).

En conclusión, las tesis doctorales sobre Artes Marciales en España son aun pocas. Más de la mitad de los autores publican en Web of Science. La mayoría de las tesis tiene nuna difusión científica notable, tanto por la cantidad de artículos que publican como por las revistas donde se publican. El autor-doctor con trabajos de investigación de calidad presenta unos índices bibliométricos aceptables para su área de conocimiento.

\section{REFERENCIAS}

AGUDELO, Diana et al. Análisis de la productividad científica de la Psicología Española a través de las tesis doctorales. Psicothema, v. 15, n. 4, p. 595-609, 2003.

ALBACETE-SÁEZ, Carlos; FUENTES-FUENTES, Mㅡㄹ del Mar. Difusión de la investigación española sobre turismo en revistas internacionales. Revista de Análisis Turístico, v. 9, n. 1, p. 14-29, 2010.

ALCAÍN, Mํㅡㄹ Dolores; CARPINTERO, Helio. La Psicología en España a través de las revistas internacionales: 1981-1999. Papeles de Psicólogo, v. 78, p. 11-20, 2001.

BOLAÑOS, Máxima. Análisis de la productividad, colaboración e impacto científico de la cardiología española (1999-2008). 2012. 206f . (Tesis doctoral)- Universidad de Valencia, Valencia.

CALVER, Michael; BRADLEY, J. Stuart. Patterns of Citations of Open Access and Non-Open Access Conservation Biology Journal Papers and Book Chapters.Conservation Biology, v. 24, n. 3, p. 872-880, 2010.

CASTILLO, Antonio; XIFRA, Jordi. Investigación bibliométrica de las tesis doctorales sobre relaciones públicas (1965/2005). Anàlisis, v. 34, p. 141-161, 2006.

CARRETERO-DIOS, Hugo; DE LOS SANTOS-ROIG, Macarena; BUELA-CASAL, Gualberto. Evaluación de la calidad de las revistas científicas de Psicología publicadas en España: consideraciones al trabajo de Alcain y Román (2005). Psicothema, v. 17, n. 4, p. 669-675, 2005.

CENTENO, Carlos et al. Tesis doctorales relacionadas con cuidados paliativos realizadas en la universidad española entre 1985 y 2000. Medicina Paliativa, v. 8, n. 4, p. 181-189, 2001. 
CLAXTON, Larry Davis. Scientific authorship. Part 2. History, recurring issues, practices, and guidelines.MutationResearch, v. 589, p. 31-45, 2005.

CURIEL-MARÍN, Elvira; FERNÁNDEZ-CANO, Antonio. Análisis cienciométrico de las tesis doctorales españolas en didáctica de las ciencias sociales (1976-2012). Revista Española de Documentación Científica, v. 38, n. 4, p. 1-10, 2015.

CRONIN, Blaise; MEHO, Lokman. Using the h-index to rank influential information scientists: Brief communication. Journal of the American Society for Information Science and Technology, v. 57, n. 9, p. 1275-1278, 2006.

DELGADO LÓPEZ-CÓZAR, Emiliano; JIMÉNEZ CONTRERAS, Evaristo; RUIZ-PÉREZ, Rafael. España y los 25 grandes de la ciencia mundial en cifras (1992-2008). Reflexiones a partir de la última actualización de Science Watch. Profesional de la Información, v. 18, n. 1, p. 81-86, 2009.

DEVÍS-DEVÍS, José et al. Disciplinas y temas de estudio en las ciencias de la actividad física y el deporte. Revista Internacional de Medicina y Ciencias de la Actividad Física y el Deporte, v. 10, n. 37, p. $150-166,2010$

DEVÍS-DEVÍS, José; VILLAMÓN, Miguel; VALENCIANO-VALCÁREL, Javier. Revistas iberoamericanas en Educación Física/Ciencias del Deporte presentes en Web of Science: evaluación y desafíos. Revista Brasileira de Ciências do Esporte, v. 36, n. 4, p.723-732, 2014.

DIEGO, Juan Ignacio; PRIM, Maria Pilar. Análisis de las tesis doctorales en otorinolaringologia presentadas en España en el período 1976-2005. Acta Otorrinolaringológica Española, v.59, n. 6,p. 292-297, 2008.

FERNÁNDEZ CANO, Antonio et al. Análisis cienciométrico de las tesis doctorales españolas en educación matemática (1976-1998). Revista Española de Documentación Científica, v. 26, n. 2, p. 162-176, 2003.

FIGUEREDO, E. Tesis doctorales Españolas sobre Anestesiología y publicaciones científicas de sus autores. Revista Española de Anestesiología y Reanimación, v. 49, n, 3, p. 124-130, 2002.

FUENTES, Eulàlia; ARGUIMBAU, Llorenç. Las tesis doctorales en España (1997-2008): análisis, estadística y repositorios cooperativos. Revista Española de Documentación Científica, v. 33, n. 1,p. 63-89, 2010.

GIL SOARES, Claudio; SARDINYA, Aline. H-Index of the Citing Articles: a Contribution to the Evaluation of Scientific Production of Experienced Researchers Revista Brasileira de Medicina do Esporte, v. 17, n. 5, p. 358-362, 2011.

GONZÁLEZ DE DIOS, Javier et al. Qué revistas médicas españolas leen y cómo se informan los médicos de atención primaria. Atención primaria, v. 43, n. 12, p. 629-636, 2011.

HERNÁNDEZ-GONZÁLEZ, Vicenç et al. Comparación entre Web of Science y Scopus. Estudio bibliometrico de las revistas de Anatomía y Morfología. International Journal of Morphology, v. 34, n. 4, p. 1369-77, 2016.

HERNÁNDEZ-GONZÁLEZ, Vicenç; REVERTER-MASIA, Joaquin; JOVÉ-DELTELL, Carme. Producción científica de los professores del àrea de Educación Física y Deportiva en Cataluña (quinquenio 2005-2009 versus 2010-2014). Sport TK- Revista Euroamericana de Ciencias del Deporte, v. 6, n. 1, p. 17-24, 2017. 
HIRSCH, Jorge. An index to quantify an individual's scientific research output. Proceedings of the National Academy of Sciences of the U.S.A., v. 102, n. 42, p. 16569-16572, 2005.

IMPERIAL, Juan; RODRÍGUEZ-NAVARRO, Alonso. La utilidad del índice $\mathbf{h}$ de Hirsch para evaluar la investigación en España. 2005. Disponible en: <http://www.bit.etsia.upm.es//mperial Rodriguez-Navarro.pdf>. Acceso en: 20 sept. 2016.

INTERNATIONAL RANKING EXPERT GROUP. Berlin principles on ranking of Higher Education Institutions, 2006. Disponible en: <https://www.che.de/downloads/Berlin Principles IREG 534.pdf>. Acceso en: 20 sept. 2016.

ISI. Citations reveal concentrated influences: Some fields have it, but what does it means, Science Watch, v.10, p.1-2, 1999.

JIMÉNEZ-CONTRERAS, Evaristo; ROBINSON-GARCÍA, Nicolás; CABEZAS-CLAVITO, Álvaro. Productividad e impacto de los investigadores españoles: umbrales de referencia por áreas científicas. Revista Española de Documentación Científica, v. 34, n. 4, p. 505-525, 2011.

MORENO-FERNÁNDEZ, Olga; MORENO-CRESPO, Pilar. Análisis bibliométrico de las tesis doctorales españolas indexadas con el descriptor "Sector de la educación" (1976/2014). Revista Española de Documentación Científica, v. 39, n. 3, p. 1-14, 2016.

OLIVAS-ÁVILA, José; MUSI-LECHUGA, Bertha. Producción en tesis doctorales de los profesores funcionarios de Psicología en España más productivos en la Web of Science. Psicothema, v. 22, n. 4, p. 917-923, 2010.

ORTEGA, Enrique et al. Estudio bibliométrico del papel de la mujer en las tesis doctorales de ciencias del deporte. Journal of Sport and Health Research, v. 7, n. 2, p. 139-148, 2015.

PALAZÓN, Maa Angeles;ORTEGA, Enrique; García-Angulo, A. Análisis bibliométrico de la producción científica en el fútbol sala. Sport TK- Revista Euroamericana de Ciencias del Deporte, v. 4, n. 2, p. 19-24, 2015.

PONCE DE LEÓN ELIZONDO, Ana; GARGALLO, Esther; LOZA, Edmundo. Análisis de las tendencias en las tesis doctorales de educación física. Cursos 1980-1981 a 1995-1996. APUNTS, Educación física, v.2, n. 52, p. 104-108, 1998.

PRICE, J. Derek. de Solla. Little Science, Big Science and beyond. Nueva York, Columbia University Press, 1986.

REPISO, Rafael; TORRES, Daniel; DELGADO, Emilio. Análisis bibliométrico y de redes sociales en tesis doctorales españolas sobre televisión (1976/2007). Revista de Educomunicación, v. 37, n. 19, p. 151-159, 2011.

REVERTER-MASIA, Joaquin et al. La produtividad científica en WoS y el índice $\mathrm{H}$ de Hirsch del área de Educación Física enEspaña yBrasil: productividad y comparación entre países. Movimento, v. 19, n. 3, p. 125-147, 2013.

REVERTER-MASIA, Joaquin et al. Disponibilidad en abierto de los artículos en Web of Science y Scopus que publican y citan los professores universitarios de Educación Física. El caso de España. Movimento, v. 21, n. 2, p. 419-433, 2015.

SALGADO, Jesús; PÁEZ, Darío. La productividad científica y el Índice h de Hirsch de la Psicología Social Española: Convergencia entre Indicadores de Productividad y Comparación con otras áreas. Psicothema, v. 19, n. 2, p.179-189, 2007. 
SIERRA, Juan Carlos; ZUBEIDAT, Ihab. Análisis bibliométrico de la revista Journal of Sex and Marital Therapy (1980-2001). Revista Internacional de Psicología Clínica y de la Salud, v. 2, n.2, p. 351-362, 2002.

TORRES, Isabel; TORRES, Daniel. Tesis doctorales sobre estudios de las mujeres en España (1976/2002): A propósito de un indicador definitivo en investigación. Revista Española de Documentación Científica, v. 28, n.4, p. 479-499, 2005. 
